\title{
Early steps in primary cilium assembly require EHD1/EHD3-dependent ciliary vesicle formation
}

Quanlong Lu, Christine Insinna, Carolyn Ott, Jimmy Stauffer, Petra A. Pintado, Juliati Rahajeng, Ulrich Baxa, Vijay Walia, Adrian Cuenca, Yoo-Seok Hwang, Ira O. Daar, Susana Lopes, Jennifer Lippincott-Schwartz, Peter K. Jackson, Steve Caplan \& Christopher J. Westlake

Nat. Cell Biol. 17, 228-240 (2015); published online 16 February 2015; corrected after print 12 March 2015

In the version of this Article originally published Fig. $3 g$ was incorrectly labelled. The top right panel should have been labelled 'EHD1' in red and the entire of $3 g$ should have been labelled ' $R a b 8 a+8 b$ siRNA' in black. The corrected image is shown below and is corrected in all online versions of the Article.

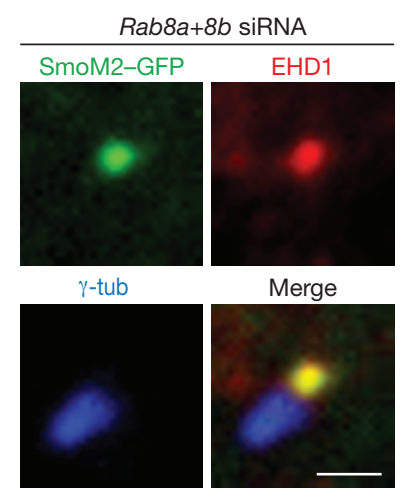

